\title{
Online Voluntary Eye Blink Detection using Electrooculogram
}

\author{
Masaki Nakanishi ${ }^{\dagger}$, Yasue Mitsukura ${ }^{\dagger}$, Yijun Wang ${ }^{\ddagger}$, Yu-Te Wang ${ }^{\ddagger}$ and Tzyy-Ping Jung ${ }^{\ddagger}$ \\ $†$ Graduate School of Science and Technology, Keio University \\ 3-14-1 Hiyoshi, Kohoku, Yokohama, Kanagawa, 223-8522 Japan \\ $¥$ Swartz Center for Computational Neuroscience, University of California, San Diego \\ La Jolla, CA, 92093 USA \\ Email: nakanishi@mitsu.sd.keio.ac.jp,mitsukura@sd.keio.ac.jp \\ Jung@sccn.ucsd.edu
}

\begin{abstract}
This paper describes the voluntary eye blink detection method using electrooculogram (EOG). There are still challenge problems to put brain-computer interface (BCI) systems to real-life applications. In general BCI systems, there is a possibility of incorrect and unintentional input because input is automatically selected even if the requirements are accidentally met. This study aims to propose the voluntary eye blink detection method and apply it to the trigger switch of BCI systems. In the proposed method, normal blink, double blink, and wink can be detected from vertical and horizontal EOG signals. We employed the positive peak of vertical and horizontal amplitude and maximum cross correlation coefficient between vertical EOG and template signal of double blink in feature extraction. Eye blinks were classified by support vector machine. As the result of simulations, an average accuracy of $97.28 \%$ was obtained using our method. In addition, the best accuracy for voluntary eye blinks was obtained for wink with accuracy of $97.69 \%$. This paper proof wink is suitable for trigger switch of BCI system, and online method for voluntary eye blink detection.
\end{abstract}

\section{Introduction}

Brain-computer interface $(\mathrm{BCI})$ realizes a direct communication between the human brain and the external environment by translating human intentions into control signals [1]. A BCI allows an individual with severe motor disabilities or aphasia to have effective control over devices such as computers, wheelchairs, assistive appliances and neural prostheses. A BCI system detects the specific patterns in a brain activity and translates these patterns into meaningful control commands. Recently, electroencephalogram (EEG)-based BCI is attracting much attention due to their noninvasiveness and high communication speed. Current EEG-based BCIs fall into four main categories such as imagery of event related synchronization (ERS) / desynchronization (ERD), event related potentials (ERP), visual evoked potentials (VEP) and slow cortical potentials (SCP) [1].

Although EEG-based BCIs have been studied for several decades, there are still challenge problems to put them to practical use. To design a practical BCI system, the follow- ing issues need to be addressed [2-7]: (1) ease of use, (2) robustness of system performance and (3) low-cost hardware and software. In general BCI systems, there is a possibility of incorrect and unintentional input because input is automatically selected even if the requirements are accidentally met. In order to solve these problems, the switch that can control input intentionally as a trigger is required. If the BCI system combined with trigger switch will be proposed, a user can input commands at their own discretion. Furthermore, false positive command transferring can be decreased.

The purpose of this study is to propose the BCI combined with trigger switch using the voluntary eye blink which is conducted intentionally. In this system, a visual feedback is presented to the user once the commands are detected. Then the user can select either input the command or ignore the command by using eye blink switch. As the first step of this study, we proposed voluntary eye blink detection method using electrooculogram (EOG). Since the duration of an eye blink is $150-250 \mathrm{~ms}$, an eye blink is suitable for a real-time application [8-10]. Voluntary eye blinks are required to be detected in distinction from involuntary eye blink, because it is hard to keep user's eye open during waiting for correct command. This study employed normal blink as involuntary blink, and double blink and wink as voluntary eye blink. This paper shows the voluntary eye blink detection and classification method using support vector machine (SVM), and online voluntary eye blink detection method.

\section{EOG signal acquisition}

\subsection{Electrical activities of eye blink}

Eye blinks are typically classified into three categories: one is a spontaneous eye blink which occurs frequently, another is a reflexive eye blink which is evoked by an external stimulus, and the other is a voluntary eye blink which is caused by intentional eye closing. An eye blink generates electrical activity in the vertical and horizontal EOG. In this study, electrical activities are detected by using four electrodes put on the upper and lower part of the dominant eye, and left and right side of each eye. The reference elec- 


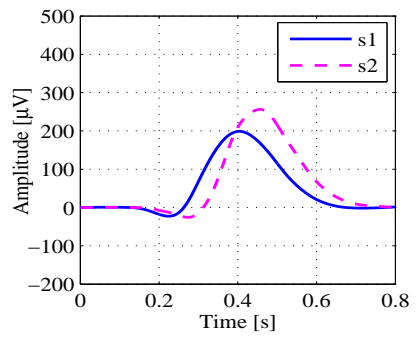

(a) Normal blink (Vertical)

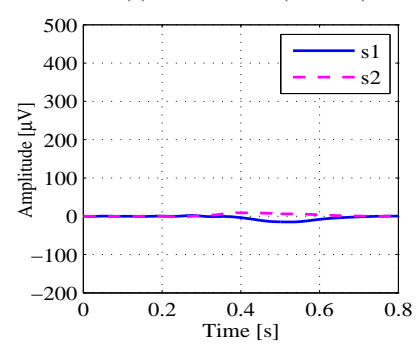

(d) Normal blink (Horizontal)

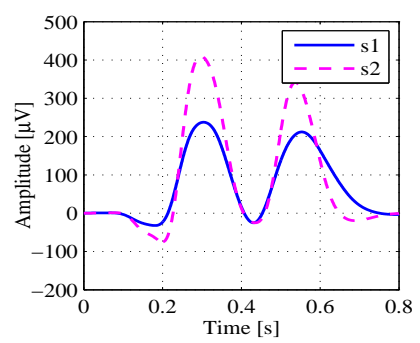

(b) Double blink (Vertical)

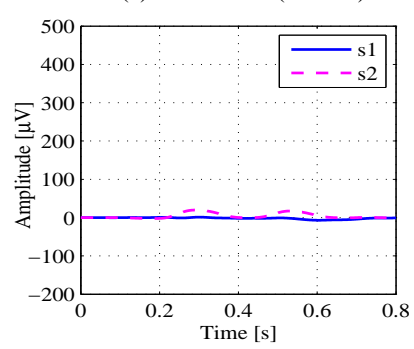

(e) Double blink (Horizontal)

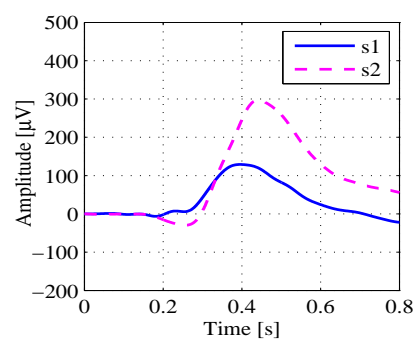

(c) Wink (Vertical)

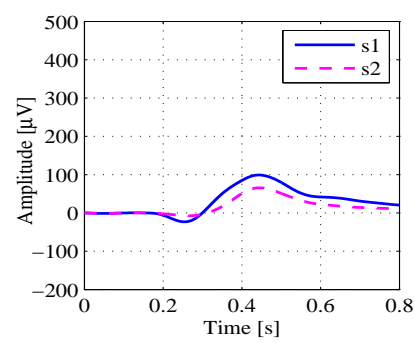

(f) Wink (Horizontal)

Figure 1: Examples of the vertical and horizontal EOG signals during (a)(d) NORMAL blink, (b)(e) DOUBLE blink and (c)(f) WINK. In these figure, s1 and s2 indicate subject number.

trode was put at the mastoid and the ground electrode was put at frontal lobe. Standard wet $\mathrm{Ag} / \mathrm{AgCl}$ electrodes were employed. The vertical signal and horizontal signal were calculated by subtracting lower part signal from upper signal, and nondominant side signal from dominant side signal respectively. EOG signals were recorded with $1000 \mathrm{~Hz}$ sampling interval.

\subsection{Behavioral tasks}

The subjects were eight healthy adult volunteers with normal or corrected-to-normal vision. The electrodes were located in the side of their dominant eye. The subjects were seated in a chair in front of the screen and asked to (1) blink binocularly as usual, (2) blink twice as soon as possible, and (3) blink monocularly at appearing fixation cross at the center of the screen. Each task is termed 'NORMAL', 'DOUBLE' and 'WINK'. The fixation cross appears for 0.5 seconds at interval of 3 seconds. In a session, the fixation cross for NORMAL blink was appeared 10 times, and then that for DOUBLE was appeared 10 times, finally that for WINK was appeared 10 times. A several-second rest was inserted between each session. The subjects were asked to repeat totally 10 sessions. The total number of trial for each eye blink was 100 for each subject.

\section{EOG signal analysis}

\subsection{Eye blink characteristics}

Figure 1 shows the example of EOG signals detected from two subjects during each task. Figure 1 (a), (b) and (c) show vertical EOG signals, and (d), (e) and (f) show horizontal EOG signals. Since each signal has the positive peak in vertical amplitude, they can be distinguishable from signals during the resting state. DOUBLE blinks are distinguishable from other eye blink because they have two peaks in vertical amplitude. WINK signals are distinguishable from other eye blinks because only horizontal signals of them have larger amplitude than those of the other task. The duration of NORMAL blinks and WINK blinks were approximately $400 \mathrm{~ms}$, and that of DOUBLE blinks were approximately $600 \mathrm{~ms}$. The amplitude of EOG signals had individual difference.

\subsection{Feature extraction}

The recorded EOG data were first preprocessed to extract $0.53-15 \mathrm{~Hz}$ signals using finite impulse response (FIR) filter. Secondly, we extracted the EOG segment of $800 \mathrm{~ms}$ from the time that the fixation cross was appeared on the screen, and EOG segment of $800 \mathrm{~ms}$ during resting state through visual inspection. In addition, we removed the segments that have obvious artifacts through visual inspection. In this study, we employed three features of eye blink signals. One is the vertical peak amplitude of EOG signals, another is the horizontal peak amplitude of EOG signals and the other is maximum cross correlation between vertical EOG signals and the template signal of DOUBLE blink prepared preliminary.

\subsection{Feature classification}

This study employed SVM to classify each eye blink using above-referenced features. SVM is one of the most popular supervised learning algorithms for solving the clas- 
Table 1: SVM result (F-score)

\begin{tabular}{cccc}
\hline \multirow{2}{*}{ Subject } & \multicolumn{2}{c}{ Kernel function } & The number of \\
\cline { 2 - 3 } & Linear & RBF & valid data \\
\hline s1 & 99.64 & 99.83 & 397 \\
s2 & 98.13 & 97.92 & 388 \\
s3 & 81.57 & 95.80 & 376 \\
s4 & 98.58 & 98.75 & 387 \\
s5 & 98.20 & 98.40 & 391 \\
s6 & 93.71 & 93.70 & 354 \\
s7 & 96.56 & 97.57 & 369 \\
s8 & 89.45 & 96.28 & 392 \\
Mean & 94.48 & 97.28 & 3054 \\
\hline
\end{tabular}

sification problems. The basic idea is to project input data onto a higher dimensional feature space by a kernel transfer function, which is easier to be separated than that in the original feature space. This study employed linear kernel and nonlinear kernel to map data onto a higher dimension space. We employed radial basis function (RBF) kernel that the highest classification accuracy was obtained after we have tried on several nonlinear kernels. 10-fold crossvalidation were applied to feature data of each subject to increase the reliability of the classification results. This study employed the F-score to measure a classification accuracy. The F-score is calculated by following equations.

$$
F=2 \cdot \frac{\text { Precision } \cdot \text { recall }}{\text { Precision }+ \text { recall }}
$$

where

$$
\begin{aligned}
\text { Precision } & =\frac{\text { True positive }}{\text { True positive }+ \text { False positive }} \\
\text { Recall } & =\frac{\text { True positive }}{\text { True positive }+ \text { False negative }}
\end{aligned}
$$

True positive and false positive indicate the proportion of data correctly and incorrectly labeled as belonging to the positive class. False negative indicate the proportion of data incorrectly labeled as belonging to negative class.

\subsection{Computer simulations}

This study shows the result of (1) classification accuracy in each subject and (2) the average confusion matrix across subjects.

Table 1 shows the F-score in each subject obtained by SVM adjusted for individual. The average F-score of 94.48 $\%$ was obtained using linear kernel and $97.28 \%$ was obtained using RBF kernel. In Six of eight subjects, higher F-score was obtained by SVM using RBF kernel than linear kernel. The highest value of F-score of $99.83 \%$ was obtained by using RBF kernel in s1 subject. F-score of s3 and s8 using linear kernel were particularly low.
Table 2: Average SVM confusion matrix across subjects

\begin{tabular}{ccccc}
\hline \multirow{2}{*}{ Input } & \multicolumn{4}{c}{ Output $(\%)$} \\
\cline { 2 - 5 } & REST & NORMAL & DOUBLE & WINK \\
\hline REST & 98.87 & 0 & 0.28 & 0.85 \\
NORMAL & 0.32 & 95.56 & 2.87 & 1.25 \\
DOUBLE & 0 & 6.58 & 92.49 & 1.49 \\
WINK & 0.54 & 0.56 & 1.21 & 97.69 \\
\hline
\end{tabular}

Table 2 shows the average confusion matrix across subjects obtained by SVM adjusted for individual. The average misclassification rate of $0.43 \%$ for REST was obtained. The better average accuracy between voluntary eye blinks was obtained for WINK (97.69\%). DOUBLE blink of 6.58 $\%$ were misclassified as NORMAL blink. This was because it was hard to blink twice quickly for some subjects. On the other hand, some subjects blinked three times when they intended to blink twice. Since we could detect WINK easier than DOUBLE, WINK is suitable for trigger switch of BCI system.

\section{Online voluntary eye blink detection}

\subsection{System description}

The proposed method was applied to online voluntary eye blink detection method. We employed DOUBLE and WINK as a voluntary eye blink, and NORMAL as an involuntary eye blink. Each eye blink can be classified by using the peak amplitude of vertical and horizontal EOG and cross correlation between vertical EOG signals and DOUBLE template as a feature value.

System operation include several functions. First, EOG signals are acquired using a $w$ millisecond moving window advancing at $s$ millisecond steps continuously. Second, vertical and horizontal EOG signals are resampled from $1000 \mathrm{~Hz}$ to $256 \mathrm{~Hz}$, and band-pass filtered at $0.53-15 \mathrm{~Hz}$. Third, feature values are calculated, and classified by using SVM with RBF kernel. Classification results can be obtained in each window, and outputs are detected only when the same result is detected in two consecutive windows. To evaluate this system, the accuracy was defined as the following equation.

$$
\text { Detection accuracy }=\frac{\text { Correct output }}{\text { Total number of output }} \times 100
$$

\section{2. system evaluation}

Figure 2 shows the detection accuracy in each subject using various parameter for a window length $w$ and a shift amount $s$. In order to reveal the trade-off between a detection accuracy and a window length or a shift amount, we 


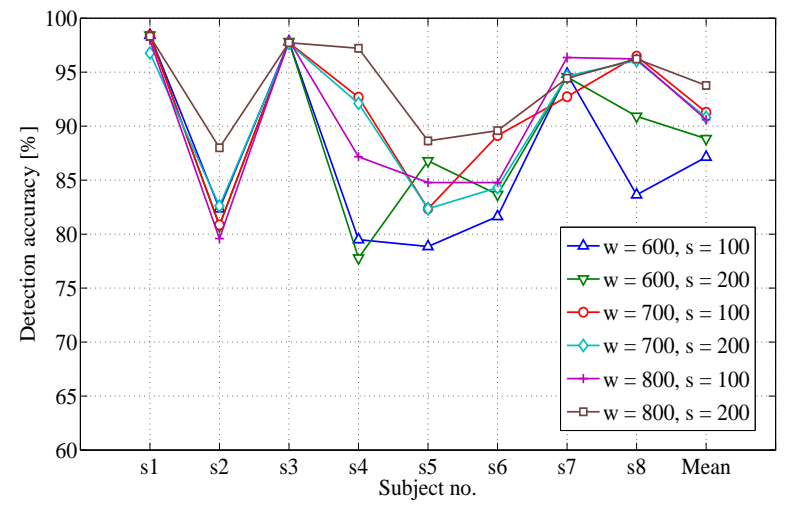

Figure 2: Online detection results

employed 600, 700, and 800 millisecond window length, and 100, 200 millisecond shift amount. As the result, the maximum accuracy of $93.77 \%$ was obtained by using 800 millisecond window and 200 millisecond shift amount. In using 600 and 700 millisecond shift amount, low accuracy was obtained because it depends on the individual difference of DOUBLE blink speed. In particular, the blink speeds of s4, s5, and s6 are actually slower than those of other subjects. Most of misclassification results were caused by confusion of NORMAL and DOUBLE, and it is considered that WINK can be detected by using proposed online method.

\section{CONCLUSIONS}

This paper presented the voluntary eye blink detection method that can classify three eye blink in distinction from resting state and online method. We employed usual blink as involuntary eye blink, and double blink and wink as voluntary eye blink. The result of the simulations showed that wink can be classified with higher accuracy than double blink. Therefore, we can find the possibility that wink is suitable for the trigger switch of BCI system. In addition, the simulation result showed that voluntary eye blinks can be detected as an online system.

Although successful result for online eye blink detection was obtained, there is still a need to improve the method. Future directions include (1)the robust method for the influence of eye movement as noise signal, and (2)the robust method for change of head pose. The future works also include development of the BCI system combined with eye blink trigger switch.

\section{References}

[1] Ali Bashashati, Mehrdad Fatourechi, Rabab K Ward and Gary E Birch, "A survey of signal processing algo- rithms in brain-computer interfaces based on electrical brain signals," Journal of Neural Engineering, Vol.4, No.2, R32-R57, 2007.

[2] Gao X, Xu D, Cheng M and Gao S, "A BCI-based environmental controller for the motion-disabled", IEEE Transaction on Neural Systems and Rehabilitation Engineering, Vol.11, Issue 2, pp.137-140, 2003.

[3] Cheng M, Gao X R, Gao S K and Xu D F, "Design and implementation of a brain-computer interface with high transfer rates", IEEE Transaction on Biomedical Engineering, Vol.49, Issue 10, pp.1181-1186, 2002.

[4] Lin C T, Ko L W, Chang M Hm Duann J R, Chen J Y, Su T P and Jung T P, "Review of wireless and wearable electroencephalogram systems and brain-computer interfaces - A Mini Review", Gerontology Vol.56, No.1, pp.112-119, 2010.

[5] Luo A and Sullivan T J, "A user-friendly SSVEP-based brain-computer interface using a time-domain classifier", Journal of Neural Engineering, Vol.7, pp.026010, 2010 .

[6] Danhua Zhu, Jordi Bieger, Gary Garcia and Ronald M. Arts, "A Survey of Stimulation Methods Used in SSVEP-Based BCIs," Journal of Computational Intelligence and Neuroscience, Vol.2010, pp.1-12, 2010.

[7] Yu-Te Wang, Yijung Wang and Tzyy-Ping Jung, "A cell-phone-based brain-computer interface for communication in daily life", Journal of Neural Engineering, Vol.8, pp.025018 (5pp), 2011.

[8] Brijil Chambayil, Rajesh Singla, R. Jha, "EEG Eye Blink Classification Using Neural Network,' in Proceedings of the World Congress on Engineering 2010 Vol. I, London, U.K., pp.2-5, 2010.

[9] Rajesh Singla, Brijil Chambayil, Arun Khosla, Jayashree Santosh, "Comparison of SVM and ANN for classification of eye events in EEG," Journal of Biomedical Science and Engineering, Vol. 4, No. 1, pp.62-69, 2011.

[10] Brijil Chambayil, Rajesh Singla, R, Jha, "Virtual Keyboard BCI using Eye blinks in EEG", in Proceedings of 2010 IEEE 6th International Conference on Wireless and Mobile Computing, Networking and Communications, Niagara Falls, ON, pp.466-470, 2010. 